\title{
Existence of Subharmonic Periodic Solutions to a Class of Second-Order Non-Autonomous Neutral Functional Differential Equations
}

\author{
Xiao-Bao Shu, ${ }^{1}$ Yongzeng Lai, ${ }^{2}$ and Fei $\mathrm{Xu}^{2}$ \\ ${ }^{1}$ Department of Mathematics, Hunan University, Changsha 410082, China \\ ${ }^{2}$ Department of Mathematics, Wilfrid Laurier University, Waterloo, ON, Canada N2L 3C5 \\ Correspondence should be addressed to Xiao-Bao Shu, sxb0221@163.com
}

Received 27 December 2011; Accepted 18 January 2012

Academic Editor: Gaston Mandata N'Guerekata

Copyright (c) 2012 Xiao-Bao Shu et al. This is an open access article distributed under the Creative Commons Attribution License, which permits unrestricted use, distribution, and reproduction in any medium, provided the original work is properly cited.

By introducing subdifferentiability of lower semicontinuous convex function $\varphi(x(t), x(t-\tau))$ and its conjugate function, as well as critical point theory and operator equation theory, we obtain the existence of multiple subharmonic periodic solutions to the following second-order nonlinear nonautonomous neutral nonlinear functional differential equation $x^{\prime \prime}(t)+x^{\prime \prime}(t-2 \tau)+f(t, x(t), x(t-$ $\tau), x(t-2 \tau))=0, x(0)=0$.

\section{Introduction}

The existence of periodic solutions for differential system has received a great deal of attention in the last few decades. Different from ordinary differential equations and partial differential equations that do not contain delay variate, it is very difficult to study the existence of periodic solutions for functional differential equations. For this reason, many mathematicians developed different approaches such as the averaging method [1], the Massera-Yoshizawa theory [2, 3], the Kaplan-York [4] method of coupled systems, the Grafton cone mapping method [5], the Nussbaum method of fixed point theory [6] and Mawhin [7] coincidence degree theory.

However, the critical point theory was rarely used in the literature, and most of the existing results were established for autonomous functional differential equations while little was done for nonautonomous equations via critical point theory. 
In this paper, by using critical point and operator equation theories, we study the existence of the following second-order nonlinear and nonautonomous mixed-type functional differential equation:

$$
\begin{gathered}
x^{\prime \prime}(t)+x^{\prime \prime}(t-2 \tau)+f(t, x(t), x(t-\tau), x(t-2 \tau))=0, \\
x(0)=0 .
\end{gathered}
$$
Section 4:

Our basic assumptions are the following $\left(A_{1}\right) \sim\left(A_{3}\right)$ and $\left(A_{4}\right) \sim\left(A_{6}\right)$ to be given in

$\left(A_{1}\right) f\left(t, x_{1}, x_{2}, x_{3}\right) \in C\left(\mathbb{R}^{4}, \mathbb{R}\right)$, and $\partial f\left(t, x_{1}, x_{2}, x_{3}\right) / \partial t \neq 0 ;$

$\left(A_{2}\right)$ there exists a continuously differentiable function $F\left(t, x_{1}, x_{2}\right) \in C^{1}\left(\mathbb{R}^{3}, \mathbb{R}\right)$ such that

$$
F_{2}^{\prime}\left(t, x_{1}, x_{2}\right)+F_{1}^{\prime}\left(t, x_{2}, x_{3}\right)=f\left(t, x_{1}, x_{2}, x_{3}\right)
$$

where $F_{2}^{\prime}\left(t, x_{1}, x_{2}\right)$ and $F_{1}^{\prime}\left(t, x_{2}, x_{3}\right)$ denote $\partial F\left(t, x_{1}, x_{2}\right) / \partial x_{2}$ and $\partial F\left(t, x_{2}, x_{3}\right) / \partial x_{2}$, respectively;

$\left(A_{3}\right) F\left(t+\tau, x_{1}, x_{2}\right)=F\left(t, x_{1}, x_{2}\right)$ for all $x_{1}, x_{2} \in \mathbb{R}$.

\section{Variational Structure}

Fix $\gamma>1, \tau>0$, where $\gamma$ is a positive integer and

$$
\begin{gathered}
H_{0}^{1}[0,2 \gamma \tau]=\left\{x(t) \in L^{2}[0,2 \gamma \tau] \mid x^{\prime} \in L^{2}[0,2 \gamma \tau], x(t) \text { is } 2 \gamma \tau \text {-periodic function in } t\right. \\
x(0)=0 \text {, and } x(t) \text { has compact support on }[0,2 \gamma \tau]\} .
\end{gathered}
$$

It is obvious that $H_{0}^{1}[0,2 \gamma \tau]$ is a Sobolev space by defining the inner product $(\cdot, \cdot)$ and the norm $\|\cdot\|$ as follows:

$$
\begin{gathered}
\langle x, y\rangle_{H_{0}^{1}[0,2 \gamma \tau]}=\int_{0}^{2 \gamma \tau} x^{\prime}(t) y^{\prime}(t) d t \\
\|x\|_{H_{0}^{1}[0,2 \gamma \tau]}=\left(\int_{0}^{2 \gamma \tau}\left|x^{\prime}(t)\right|^{2} d t\right)^{1 / 2}, \quad \forall x, y \in H_{0}^{1}[0,2 \gamma \tau] .
\end{gathered}
$$

Moreover, $x(t) \in H_{0}^{1}[0,2 \gamma \tau]$ can be expressed as

$$
x(t)=a_{0}+\sum_{k=1}^{\infty}\left(a_{k} \cos \frac{k \pi}{\gamma \tau} t+b_{k} \sin \frac{k \pi}{\gamma \tau} t\right) .
$$


Let us consider the functional $I(x)$ defined on $H_{0}^{1}[0,2 \gamma \tau]$ by

$$
I(x)=\int_{0}^{2 \gamma \tau}\left[x^{\prime}(t) x^{\prime}(t-\tau)-F(t, x(t), x(t-\tau))\right] d t .
$$

For all $x, y \in H_{0}^{1}[0,2 \gamma \tau]$ and $\varepsilon>0$, we know that

$$
\begin{aligned}
I(x+\varepsilon y)= & I(x)+\varepsilon\left(\int _ { 0 } ^ { 2 \gamma \tau } \left[x^{\prime}(t) y^{\prime}(t-\tau)+x^{\prime}(t-\tau) y^{\prime}(t)\right.\right. \\
& \quad-(F(t, x(t)+\varepsilon y(t), x(t-\tau)+\varepsilon y(t-\tau))-F(t, x(t), x(t-\tau)))] d t) \\
& +\varepsilon^{2} \int_{0}^{2 \gamma \tau} y^{\prime}(t) y^{\prime}(t-\tau) d t .
\end{aligned}
$$

It is then easy to see that

$$
\begin{gathered}
\left\langle I^{\prime}(x), y\right\rangle=\int_{0}^{2 \gamma \tau}\left[x^{\prime}(t) y^{\prime}(t-\tau)+x^{\prime}(t-\tau) y^{\prime}(t)-F_{1}^{\prime}(t, x(t), x(t-\tau)) y(t)\right. \\
\left.-F_{2}^{\prime}(t, x(t), x(t-\tau)) y(t-\tau)\right] d t
\end{gathered}
$$

where $I^{\prime}(x)$ denotes the Frechet differential of the function $I(x)$. By the periodicity of $F\left(t, u_{1}, u_{2}\right), x(t)$, and $y(t)$, we have

$$
\begin{aligned}
\int_{0}^{2 \gamma \tau} x^{\prime}(t) y^{\prime}(t-\tau) d t & =\int_{0}^{2 \gamma \tau} x^{\prime}(t) d y(t-\tau)=\left.x^{\prime}(t) y(t-\tau)\right|_{0} ^{2 \gamma \tau}, \\
-\int_{0}^{2 \gamma \tau} x^{\prime \prime}(t) y(t-\tau) d t & =-\int_{0}^{2 \gamma \tau} x^{\prime \prime}(t+\tau) y(t) d t, \\
\int_{0}^{2 \gamma \tau} x^{\prime}(t-\tau) y^{\prime}(t) d t & =-\int_{0}^{2 \gamma \tau} x^{\prime \prime}(t-\tau) y(t) d t .
\end{aligned}
$$

Similarly, we have

$$
\begin{aligned}
\int_{0}^{2 \gamma \tau} F_{2}^{\prime}(t, x(t), x(t-\tau)) y(t-\tau) d t & =\int_{-\tau}^{(2 \gamma-1) \tau} F_{2}^{\prime}(t+\tau, x(t+\tau), x(t)) y(t) d t \\
& =\int_{0}^{2 \gamma \tau} F_{2}^{\prime}(t, x(t+\tau), x(t)) y(t) d t
\end{aligned}
$$


Hence,

$$
\begin{aligned}
\left\langle I^{\prime}(x), y\right\rangle=\int_{0}^{2 \gamma \tau}[ & -x^{\prime \prime}(t+\tau)-x^{\prime \prime}(t-\tau) \\
& \left.\quad-F_{u_{1}}^{\prime}(t, x(t) x(t-\tau))-F_{u_{2}}^{\prime}(t, x(t+\tau), x(t))\right] y(t) d t .
\end{aligned}
$$

Therefore, the Euler equation corresponding to the functional $I(x)$ is

$$
x^{\prime \prime}(t+\tau)+x^{\prime \prime}(t-\tau)+\left[F_{u_{1}}^{\prime}(t, x(t), x(t-\tau))+F_{u_{2}}^{\prime}(t, x(t+\tau), x(t))\right]=0 .
$$

It is not difficult to see that (2.10) is equivalent to (1.1). Thus, system (1.1) is the Euler equation of the functional $I(x)$. It follows that it is possible to obtain $2 \gamma \tau$-periodic solutions of system (1.1) by seeking critical points of the functional $I(x)$.

Since $I(x)$ has neither a supremum nor an infimum, we do not seek critical points of the functional $I(x)$ by the extremum method. But we may use operator equation theory. First via the dual variational principle, we obtain new operator equations (see (4.16)) related to (1.1). Then solutions to system (1.1) are obtained by seeking critical points of operator equation (4.16).

In this paper, our main tool is the following.

Lemma 2.1 (Maintain Pass Theorem). Let $H$ be a real Banach space. If $I(\cdot) \in C^{1}(H, \mathbb{R})$ satisfies the Palais-Smale condition as well as the following additional conditions:

(1) there exist constants $\rho>0$ and $a>0$ such that $I(x) \geq a$, for all $x \in \partial B_{\rho}$, where $B_{\rho}=$ $\left\{x \in H:\|x\|_{H}<\rho\right\}$,

(2) $I(\theta) \leq 0$ and there exists $x_{0} \bar{\epsilon} B_{\rho}$ such that $I\left(x_{0}\right) \leq 0$, then $c=\inf _{h \in \Gamma} \sup _{s \in[0,1]} I(h(s))$ is a positive critical value of $I$, where

$$
\Gamma=\left\{h \in C([0,1], H) \mid h(0)=\theta, h(1)=x_{0}\right\} .
$$

The rest of this paper is organized as follows. Subdifferentiability of lower semicontinuous convex function $\varphi(x(t), x(t-\tau))$ and its conjugate function are introduced in Section 3. In Section 4, we first give the definition of the weak solution to (1.1), then we establish the new operator equation (4.16) related to (1.1) by the conjugate function of $F(t, x(t), x(t-\tau))$ and show that we can obtain solution to (1.1) from the solution to operator equation (4.16). In Section 5, by seeking critical points of operator equation (4.16), we obtain the result that there exist multiple subharmonic periodic solutions to system (1.1). Finally in Section 6, an example and a remark are given to illustrate our result.

\section{The Subdifferentiability and the Conjugate Function of the Lower Semicontinuous Convex Function $\varphi(x(t), x(t-\tau))$}

Let $X$ be a space of all given $n \times \tau$-periodic functions in $t$ and a Banach space, where $n \in N$ is a positive integer. Denote $\overline{\mathbb{R}}=\mathbb{R} \cup\{+\infty\}$. Let $\varphi: X^{2} \rightarrow \overline{\mathbb{R}}$ be a lower semicontinuous 
convex function. Generally, $\varphi$ is not always differentiable in conventional sense, but we may generalize the definition of "derivative" as follows.

Definition 3.1. Let $\left(x_{1}^{*}, x_{2}^{*}\right) \in X^{*} \times X^{*}$. We say that $\left(x_{1}^{*}, x_{2}^{*}\right)$ is a subgradient of $\varphi$ at point $\left(x_{0}(t), x_{0}(t-\tau)\right) \in X \times X$ if

$$
\varphi\left(x_{0}(t), x_{0}(t-\tau)\right)+\left\langle x_{1}^{*}, x(t)-x_{0}(t)\right\rangle+\left\langle x_{2}^{*}, x(t-\tau)-x_{0}(t-\tau)\right\rangle \leq \varphi(x(t), x(t-\tau)) .
$$

For all $x_{0}(t) \in X$, the set of all subgradients of $\varphi$ at point $\left(x_{0}(t), x_{0}(t-\tau)\right)$ will be called the sub-differential of $\varphi$ at point $\left(x_{0}(t), x_{0}(t-\tau)\right)$ and will be denoted by $\partial \varphi\left(x_{0}(t), x_{0}(t-\tau)\right)$.

By the definition of Subdifferentiability of function $\varphi$, we may define its conjugate function $\varphi^{*}$ by

$$
\varphi^{*}\left(x_{1}^{*}, x_{2}^{*}\right)=\sup \left\{\left\langle x_{1}^{*}, x(t)\right\rangle+\left\langle x_{2}^{*}, x(t-\tau)\right\rangle-\varphi(x(t), x(t-\tau))\right\}
$$

where $\langle\cdot\rangle$ denotes the duality relation of $X^{*}$ and $X$. So it is not difficult to obtain the following propositions.

Proposition 3.2. $\varphi^{*}$ is a lower semicontinuous convex function ( $\varphi^{*}$ may have functional value $+\infty$, but not functional value $-\infty$ ).

Proposition 3.3. If $\varphi \leq \psi$, then $\varphi^{*} \geq \psi^{*}$.

Proposition 3.4 (Yang inequality). One has

$$
\varphi(x(t), x(t-\tau))+\varphi^{*}\left(x_{1}^{*}, x_{2}^{*}\right) \geq\left\langle x_{1}^{*}, x(t)\right\rangle+\left\langle x_{2}^{*}, x(t-\tau)\right\rangle .
$$

Proposition 3.5. One has

$$
\begin{aligned}
\varphi(x(t), x(t-\tau))+\varphi^{*}\left(x_{1}^{*}, x_{2}^{*}\right) & =\left\langle x_{1}^{*}, x(t)\right\rangle+\left\langle x_{2}^{*}, x(t-\tau)\right\rangle \\
& \Longleftrightarrow\left(x_{1}^{*}, x_{2}^{*}\right) \in \partial \varphi(x(t), x(t-\tau)) .
\end{aligned}
$$

Proposition 3.6. $\varphi^{*}$ does not always equal $+\infty$.

Proof. Let $x_{0}(t) \in X$ and $\beta \in \mathbb{R}$ such that $\varphi\left(x_{0}(t), x_{0}(t-\tau)\right)<+\infty$ and $\beta_{0}<\varphi\left(x_{0}(t), x_{0}(t-\tau)\right)$. We consider the two convex sets in $X^{2} \times \mathbb{R}$ defined by

$$
\begin{aligned}
& A=\operatorname{epi} \varphi \triangleq\left\{(x(t), x(t-\tau), \beta) \in X^{2} \times \mathbb{R} \mid: \varphi(x(t), x(t-\tau))<+\infty, \beta>\varphi(x(t), x(t-\tau))\right\} \\
& B=\left\{\left(x_{0}(t), x_{0}(t-\tau), \beta_{0}\right)\right\} .
\end{aligned}
$$


By Hahn-Banach Theorem, we know that there exists $\left(f_{1}, f_{2}, k\right) \in X^{*} \times X^{*} \times \mathbb{R}$ and $\alpha \in \mathbb{R}$ such that

$$
\begin{aligned}
& \left\langle f_{1}, x(t)\right\rangle+\left\langle f_{2}, x(t-\tau)\right\rangle+k \beta>\alpha, \quad \forall(x(t), x(t-\tau), \beta) \in \operatorname{eip} \varphi, \\
& \left\langle f_{1}, x_{0}(t)\right\rangle+\left\langle f_{2}, x_{0}(t-\tau)\right\rangle+k \beta_{0}<\alpha .
\end{aligned}
$$

So, we have

$$
\left.\left\langle f_{1}, x_{0}(t)\right\rangle+\left\langle f_{2}, x_{0}(t-\tau)\right\rangle+k \varphi\left(x_{0}(t), x_{0}(t-\tau)\right)>\alpha\right\rangle\left\langle f_{1}, x_{0}(t)\right\rangle+\left\langle f_{2}, x_{0}(t-\tau)\right\rangle+k \beta_{0} .
$$

Thus $k>0$, and

$$
\left\langle-\frac{1}{k} f_{1}, x(t)\right\rangle+\left\langle-\frac{1}{k} f_{2}, x(t-\tau)\right\rangle-\varphi(x(t), x(t-\tau))<-\frac{\alpha}{k}
$$

Since $\varphi^{*}$ is a lower semicontinuous convex function that does not always equal $+\infty$, we may define its conjugate function $\varphi^{* *}$ by

$$
\varphi^{* *}\left(x_{1}^{* *}, x_{2}^{* *}\right)=\sup _{\left(x_{1}^{*}, x_{2}^{*}\right) \in X^{*} \times X^{*}}\left\{\left\langle x_{1}^{* *}, x_{1}^{*}\right\rangle+\left\langle x_{2}^{* *}, x_{2}^{*}\right\rangle-\varphi\left(x_{1}^{*}, x_{2}^{*}\right)\right\}
$$

where $\langle\cdot\rangle$ denotes the duality relation of $X^{* *}$ and $X^{*}$.

Theorem 3.7. Let $\varphi$ be a lower semicontinuous convex function that does not always equal $+\infty$, then $\varphi^{* *}=\varphi$.

Proof. We divide our proof into two parts. First we show that $\varphi^{* *}=\varphi$ holds when $\varphi>0$ and then $\varphi^{* *}=\varphi$ holds for all lower semicontinuous convex functions $\varphi$ that do not always equal $+\infty$.

(i) The case when $\varphi>0$.

From the definition of $\varphi^{* *}$ and Yang inequality, it is obvious that $\varphi^{* *} \leq \varphi$ holds.

Next, to prove $\varphi^{* *} \geq \varphi$ holds, suppose to the contrary that there exist a point $\left(x_{0}(t), x_{0}(t-\tau)\right) \in X^{2}$, such that $\varphi^{* *}\left(x_{0}(t), x_{0}(t-\tau)\right)<\varphi\left(x_{0}(t), x_{0}(t-\tau)\right)$ holds.

Consider the two convex sets

$$
\begin{aligned}
& A=\operatorname{eip} \varphi \triangleq\left\{(x(t), x(t-\tau), \beta) \in X^{2} \times \mathbb{R} \mid \varphi(x(t), x(t-\tau))<+\infty, \beta \geq \varphi(x(t), x(t-\tau))\right\} \\
& B_{0}=\left\{\left(x_{0}(t), x_{0}(t-\tau), \varphi^{* *}\left(x_{0}(t), x_{0}(t-\tau)\right)\right)\right\} .
\end{aligned}
$$

By the Hahn-Banach Theorem, we know that there exist $\left(g_{1}, g_{2}, k^{*}\right) \in X^{*} \times X^{*} \times \mathbb{R}$ and $\alpha_{1} \in \mathbb{R}$ such that

$$
\begin{gathered}
\left\langle g_{1}, x(t)\right\rangle+\left\langle g_{2}, x(t-\tau)\right\rangle+k^{*} \beta>\alpha_{1}, \quad \forall(x(t), x(t-\tau), \beta) \in \operatorname{eip} \varphi \\
\left\langle g_{1}, x_{0}(t)\right\rangle+\left\langle g_{2}, x_{0}(t-\tau)\right\rangle+k^{*} \varphi^{* *}\left(x_{0}(t), x_{0}(t-\tau)\right)<\alpha_{1}
\end{gathered}
$$


So, it follows that $k^{*} \geq 0$. Let $\varepsilon>0$. Using $\varphi>0$ and (3.11), one obtains that

$$
\left\langle g_{1}, x(t)\right\rangle+\left\langle g_{2}, x(t-\tau)\right\rangle+\left(k^{*}+\varepsilon\right) \varphi(x(t), x(t-\tau)) \geq \alpha_{1}, \quad \forall(x(t), x(t-\tau)) \in D(\varphi) .
$$

Thus, we have

$$
\varphi^{*}\left(-\frac{g_{1}}{k^{*}+\varepsilon},-\frac{g_{2}}{k^{*}+\varepsilon}\right) \leq-\frac{\alpha_{1}}{k^{*}+\varepsilon}
$$

Then, by the definition of $\varphi^{* *}$, we obtain that

$$
\begin{aligned}
\varphi^{* *}\left(x_{0}(t), x_{0}(t-\tau)\right) & \geq\left\langle-\frac{g_{1}}{k^{*}+\varepsilon}, x_{0}(t)\right\rangle+\left\langle-\frac{g_{2}}{k^{*}+\varepsilon}, x_{0}(t-\tau)\right\rangle-\varphi^{*}\left(-\frac{g_{1}}{k^{*}+\varepsilon},-\frac{g_{2}}{k^{*}+\varepsilon}\right) \\
& \geq\left\langle-\frac{g_{1}}{k^{*}+\varepsilon}, x_{0}(t)\right\rangle+\left\langle-\frac{g_{2}}{k^{*}+\varepsilon}, x_{0}(t-\tau)\right\rangle+\frac{\alpha_{1}}{k^{*}+\varepsilon}
\end{aligned}
$$

That is to say,

$$
\left\langle g_{1}, x_{0}(t)\right\rangle+\left\langle g_{2}, x_{0}(t-\tau)\right\rangle+\left(k^{*}+\varepsilon\right) \varphi^{* *}\left(x_{0}(t), x_{0}(t-\tau)\right) \geq \alpha_{1}, \quad \forall \varepsilon>0,
$$

which is a contradiction to (3.12).

(ii) For all $\varphi$, by Proposition 3.6, we know that $D\left(\varphi^{*}\right) \neq \emptyset$. Choose $\left(x_{10}^{*}, x_{20}^{*}\right) \in D\left(\varphi^{*}\right)$, and define function $\bar{\varphi}$ by

$$
\bar{\varphi}(x(t), x(t-\tau))=\varphi(x(t), x(t-\tau))-\left\langle x_{10}^{*}, x(t)\right\rangle-\left\langle x_{20}^{*}, x(t-\tau)\right\rangle+\varphi^{*}\left(x_{10}^{*}, x_{20}^{*}\right) .
$$

Then $\bar{\varphi}$ is a lower semicontinuous convex function that is not always equal to $+\infty$ and satisfies $\bar{\varphi} \geq 0$. By the result of (i), we have that $\bar{\varphi}^{* *}=\bar{\varphi}$. On the other hand, we have

$$
\begin{aligned}
\bar{\varphi}^{*}\left(x_{1}^{*}, x_{2}^{*}\right) & =\varphi^{*}\left(x_{1}^{*}+x_{10}^{*}, x_{2}^{*}+x_{20}^{*}\right)-\varphi^{*}\left(x_{10}^{*}, x_{20}^{*}\right), \\
\bar{\varphi}^{* *}(x(t), x(t-\tau)) & =\bar{\varphi}(x(t), x(t-\tau))-\left\langle x_{10}^{*}, x(t)\right\rangle-\left\langle x_{20}^{*}, x(t-\tau)\right\rangle+\varphi^{*}\left(x_{10}^{*}, x_{20}^{*}\right) .
\end{aligned}
$$

That is, $\bar{\varphi}^{* *}=\varphi$.

Corollary 3.8. Let $\varphi$ be a lower semicontinuous convex function that is not always equal to $+\infty$. Then $\left(x_{1}^{*}, x_{2}^{*}\right) \in \partial \varphi(x(t), x(t-\tau))$ if and only if

$$
(x(t), x(t-\tau)) \in \partial \varphi^{*}\left(x_{1}^{*}, x_{2}^{*}\right) .
$$


Proof. One has

$$
\begin{aligned}
\left(x_{1}^{*}, x_{2}^{*}\right) \in \partial \varphi(x(t), x(t-\tau)) & \Longleftrightarrow\left\langle x_{1}^{*}, x(t)\right\rangle+\left\langle x_{2}^{*}, x(t-\tau)\right\rangle \\
& =\varphi(x(t), x(t-\tau))+\varphi^{*}\left(x_{1}^{*}, x_{2}^{*}\right) \\
& =\varphi^{* *}(x(t), x(t-\tau))+\varphi^{*}\left(x_{1}^{*}, x_{2}^{*}\right) \\
& \Longleftrightarrow(x(t), x(t-\tau)) \in \partial \varphi^{*}\left(x_{1}^{*}, x_{2}^{*}\right) .
\end{aligned}
$$

\section{Weak Solution to (1.1)}

Define an operator $A=d^{2} / d t^{2}$. By (2.6) and

$$
\begin{aligned}
\langle u(t), A(\omega(t))\rangle & =\int_{0}^{2 \gamma \tau} u(t) \omega^{\prime \prime}(t) d t=\left.u(t)\left(\omega^{\prime}(t)\right)\right|_{0} ^{2 \gamma \tau}-\int_{0}^{2 \gamma \tau} \omega^{\prime}(t) u^{\prime}(t) d t \\
& =-\int_{0}^{2 \gamma \tau} u^{\prime}(t) d \omega(t)=-\left.u^{\prime}(t) \omega(t)\right|_{0} ^{2 \gamma \tau}+\int_{0}^{2 \gamma \tau} \omega(t) d u^{\prime}(t) \\
& =\langle A u(t), \omega(t)\rangle
\end{aligned}
$$

as well as

$$
\langle u(t), A(\omega(t-\tau))\rangle=\langle A u(t+\tau), \omega(t)\rangle, \quad\langle u(t-\tau), A(\omega(t))\rangle=\langle A(u(t-\tau)), \omega(t)\rangle
$$

we may define a weak solution to (1.1) as follows.

Definition 4.1. For $u \in L^{p}[0,2 \gamma \tau]$, we say that $u$ is a weak solution to (1.1), if

$$
\begin{aligned}
& \langle u(t), A(\omega(t-\tau))\rangle+\langle u(t-\tau), A(\omega(t))\rangle+\left\langle\omega(t), F_{1}^{\prime}(t, u(t), u(t-\tau))\right\rangle \\
& \quad+\left\langle\omega(t-\tau), F_{2}^{\prime}(t, u(t), u(t-\tau))\right\rangle=0
\end{aligned}
$$

for all $\omega(t) \in D(A) \cap L^{p}[0,2 \gamma \tau]$, where

$$
\langle u(t), v(t)\rangle=\int_{0}^{2 \gamma \tau} u(t) v(t) d t
$$

when $u(t) \in L^{p}[0,2 \gamma \tau], v(t) \in L^{q}[0,2 \gamma \tau]$, where $2<p<+\infty$, $(1 / p)+(1 / q)=1$. 
Our objective is to define the conjugate function of $F(t, x(t), x(t-\tau))$ by using the definition of Subdifferentiability of lower semicontinuous convex function and by making use of the dual variational structure. So we add three more conditions on function $F(t, x(t), x(t-\tau))$ as follows:

$\left(A_{4}\right) u=\left(u_{1}, u_{2}\right) \rightarrow F\left(t, u_{1}, u_{2}\right)$ is a continuously differentiable and strictly convex function and satisfies

$$
F(t, 0,0)=0, \quad F_{1}^{\prime}(t, 0,0)=F_{2}^{\prime}(t, 0,0)=0 \quad \forall t \in[0,2 \gamma \tau]
$$

$\left(A_{5}\right)$ for $\alpha_{2}=1 / p$, there exist constants $M, C>0$, such that when $|u|=\sqrt{u_{1}^{2}+u_{2}^{2}} \geq C$ we have

$$
\begin{aligned}
& F\left(t, u_{1}, u_{2}\right) \leq \alpha_{2}\left[F_{1}^{\prime}\left(t, u_{1}, u_{2}\right) u_{1}+F_{2}^{\prime}\left(t, u_{1}, u_{2}\right) u_{2}\right] \\
& F\left(t, u_{1}, u_{2}\right) \leq M|u|^{\left(1 / \alpha_{2}\right)}
\end{aligned}
$$

$\left(A_{6}\right)$

$$
\lim _{|u| \rightarrow 0} \frac{F\left(t, u_{1}, u_{2}\right)}{|u|^{2}}=0
$$

The conjugate function of function $F(t, x(t), x(t-\tau))$ is defined by

$$
H(t, \omega(t), \omega(t-\tau))=\sup _{x(t) \in L^{p}[0,2 \gamma \tau]}\{\langle\omega(t), x(t)\rangle+\langle\omega(t-\tau), x(t-\tau)\rangle-F(t, x(t), x(t-\tau))\},
$$

for $t \in[0,2 \gamma \tau]$.

Then $H$ is a continuously differentiable and strictly convex function. By the duality principle (Corollary 3.8), we have that

$$
\begin{aligned}
(\omega(t), \omega(t-\tau)) & =\left(F_{1}^{\prime}(t, x(t), x(t-\tau)), F_{2}^{\prime}(t, x(t), x(t-\tau))\right) \\
& \Longleftrightarrow\left(H_{1}^{\prime}(t, \omega(t), \omega(t-\tau)), H_{2}^{\prime}(t, \omega(t), \omega(t-\tau))\right)=(x(t), x(t-\tau)),
\end{aligned}
$$

where $H_{1}^{\prime}(t, \omega(t), \omega(t-\tau))$ and $H_{2}^{\prime}(t, \omega(t), \omega(t-\tau))$ denote $\partial H(t, \omega(t), \omega(t-\tau)) / \partial \omega(t)$ and $\partial H(t, \omega(t), \omega(t-\tau)) / \partial \omega(t-\tau)$, respectively.

Example 4.2. Let $F(x(t), x(t-\tau))=(1 / p)\left({\sqrt{x^{2}(t)+x^{2}(t-\tau)}}^{p}\right.$. Then

$$
H(\omega(t), \omega(t-\tau))=\frac{1}{q}\left(\sqrt{\omega^{2}(t)+\omega^{2}(t-\tau)}\right)^{q} .
$$


Proof. The above expression holds since

$$
\begin{aligned}
H(\omega(t), \omega(t-\tau)) & =\sup _{x(t) \in L^{p}[0,2 \gamma \tau]}\left\{\langle\omega(t), x(t)\rangle+\langle\omega(t-\tau), x(t-\tau)\rangle-\frac{1}{p}\left(\sqrt{x^{2}(t)+x^{2}(t-\tau)}\right)^{p}\right\} \\
& =\sup _{\lambda>0}\left\{\sqrt{\omega^{2}(t)+\omega^{2}(t-\tau)} \lambda-\frac{1}{p} \lambda^{p}\right\} \\
& =\frac{1}{q}\left(\sqrt{\omega^{2}(t)+\omega^{2}(t-\tau)}\right)^{q} .
\end{aligned}
$$

Let $R(A)$ denote the value field of operator $A$. Then $R(A)$ is a closed set. Let $P$ be the orthogonal projection operator of $R(A)$ and $\widehat{K}=A^{-1} P$. Then it is not difficult to see that $\widehat{K}$ maps continuous continuation into a compact operator of $L^{q}[0,2 \gamma \tau] \rightarrow L^{q}[0,2 \gamma \tau]$.

Let

$$
\begin{aligned}
E & =\left\{(v(t), v(t-\tau)) \in\left(L^{q}[0,2 \gamma \tau]\right)^{2} v(0)=0 \mid\langle\phi(t), v(t)\rangle=\langle\phi(t), v(t-\tau)\rangle\right. \\
& \left.=\langle\phi(t-\tau), v(t)\rangle=0, \quad \forall \phi(t) \in \mathfrak{R}(A) \cap L^{p}[0,2 \gamma \tau], \phi(0)=0\right\},
\end{aligned}
$$

where $\mathfrak{R}(A)=\{u \in D(A) \mid A(u(t)+u(t-2 \tau))=0\}$.

Remark 4.3. In fact, for all $x(0)=0, x(t) \in L^{p}[0,2 \gamma \tau]$, or $x(t) \in L^{q}[0,2 \gamma \tau], x(t)$ can be expressed as

$$
x(t)=a_{0}+\sum_{k=1}^{\infty}\left(a_{k} \cos \frac{k \pi}{r \tau} t+b_{k} \sin \frac{k \pi}{r \tau} t\right) .
$$

So, it follows that

$$
\langle v(t), \phi(t)\rangle=0 \Longleftrightarrow\langle\phi(t-\tau), v(t)\rangle=\langle\phi(t), v(t-\tau)\rangle=\langle\phi(t), v(t)\rangle=0 .
$$

Thus $E$ can also be expressed as

$$
\begin{gathered}
E=\left\{(v(t), v(t-2 \tau)) \in L^{q}[0,2 \gamma \tau] \times L^{q}[0,2 \gamma \tau], v(0)=0 \mid\langle\phi(t), v(t)\rangle=0,\right. \\
\left.\forall \phi(t) \in \mathfrak{R}(A) \cap L^{p}[0,2 \gamma \tau], \phi(0)=0\right\} .
\end{gathered}
$$


We want $\{(v(t), v(t-\tau)),(\chi(t), \chi(t-\tau))\}$ to satisfy

$$
\begin{aligned}
& x(t)=\widehat{K}(v(t-\tau))+H_{1}^{\prime}(t, v(t), v(t-\tau)), \\
& x(t-\tau)=\widehat{K}(v(t))+H_{2}^{\prime}(t, v(t), v(t-\tau)),
\end{aligned}
$$

where $(v(t), v(t-\tau)) \in E, \chi(t) \in \mathfrak{R}(A) \cap L^{p}[0,2 \gamma \tau]$, that is, $(\chi(t), \chi(t-\tau)) \in E^{\perp}$.

If $\{(v(t), v(t-\tau)),(\chi(t), \chi(t-\tau))\}$ is a solution to (4.16) and if $u(t)=H_{1}^{\prime}(t, v(t), v(t-$ $\tau)), u(t-\tau)=H_{2}^{\prime}(t, v(t), v(t-\tau))$, then by the duality principle and (4.16), we have

$$
\begin{aligned}
&\langle u(t), A(z(t-\tau))\rangle+\langle u(t-\tau), A(z(t))\rangle+\left\langle z(t), F_{1}^{\prime}(t, u(t), u(t-\tau))\right\rangle+\left\langle z(t-\tau), F_{2}^{\prime}(t, u(t), u(t-\tau))\right\rangle \\
&=\left\langle H_{1}^{\prime}\left(t, v(t), v^{\prime}(t-\tau)\right), A(z(t-\tau))\right\rangle+\left\langle H_{2}^{\prime}(t, v(t), v(t-\tau)), A(z(t))\right\rangle+\langle z(t), v(t)\rangle \\
&+\langle z(t-\tau), v(t-\tau)\rangle \\
&=\langle x(t)-\widehat{K}(v(t-\tau)), A(z(t-\tau))\rangle+\langle x(t-\tau)-\widehat{K}(v(t)), A(z(t))\rangle+\langle z(t), v(t)\rangle \\
&+\langle z(t), v(t)\rangle \\
&=-\langle v(t), z(t)\rangle-\langle v(t), z(t)\rangle+\langle z(t), v(t)\rangle+\langle z(t), v(t)\rangle=0 \quad \forall z(t) \in D(A) \cap L^{p}[0,2 \gamma \tau] .
\end{aligned}
$$

Thus, $u(t)$ is a weak solution to (1.1).

\section{Existence of Solutions to Operator Equation (4.16)}

In this section, we discuss the existence of solutions to operator equation (4.16) by using the critical point theory. Our main result is the following.

Theorem 5.1. Under assumptions $\left(A_{1}\right) \sim\left(A_{6}\right)$, problem (1.1) has at least one nontrivial weak $2 \gamma \tau$ periodic solution.

To prove this theorem, we state and prove the following lemmas first.

Let $v=(v(t), v(t-\tau))$, and

$$
K\left(\begin{array}{c}
v(t) \\
v(t-\tau)
\end{array}\right)=\left(\begin{array}{cc}
0 & \widehat{K} \\
\widehat{K} & 0
\end{array}\right)\left(\begin{array}{c}
v(t) \\
v(t-\tau)
\end{array}\right)=\left(\begin{array}{c}
\widehat{K} v(t-\tau) \\
\widehat{K} v(t)
\end{array}\right) .
$$

It is not difficult to verify that $\langle K(v), \psi\rangle=\langle v, K(\psi)\rangle=\langle\bar{K} v(t-\tau), \psi(t)\rangle+\langle\bar{K} v(t), \psi(t-$ $\tau)\rangle$, where $\psi=(\psi(t), \psi(t-\tau))$, that is, the operator $K$ is a symmetric operator. 

by

We may obtain solutions to (4.16) by seeking critical points of functional $J(v)$ defined

$$
\begin{aligned}
J(v)= & \frac{1}{2}\langle K(v), v\rangle+\int_{0}^{2 \gamma \tau} H(t, v) d t \\
= & \frac{1}{2}\langle\widehat{K} v(t-\tau), v(t)\rangle+\frac{1}{2}\langle\widehat{K} v(t), v(t-\tau)\rangle \\
& +\int_{0}^{2 \gamma \tau} H(t, v(t), v(t-\tau)) d t \quad \forall(v(t), v(t-\tau)) \in E .
\end{aligned}
$$

$J$ may be regarded as the restriction to $E$ of function $\widehat{J}$ defined on $L^{q}[0,2 \gamma \tau] \times L^{q}[0,2 \gamma \tau]$ since both functions have identical component on $E$. Moreover

$$
\widehat{J}^{\prime}(v)=K(v)+H^{\prime}(v)
$$

Since

$$
\left\langle\widehat{J}^{\prime}(v)-J^{\prime}(v), z\right\rangle=0, \quad \forall v \in E, z=(z(t), z(t-\tau)) \in E,
$$

there exist $\chi(t) \in \mathfrak{R}(A)$ and $\chi_{v}=\left(\chi_{v}(t), \chi_{v}(t-\tau)\right) \in E^{\perp}$ such that

$$
\widehat{J}^{\prime}(v)-J^{\prime}(v)=x_{v}
$$

So if $v^{*}$ is a critical point of $J^{\prime}\left(v^{*}\right)=0$ on $E$, then there exists $\chi_{v^{*}}^{*}=\left(\chi_{v^{*}}^{*}(t), \chi_{v^{*}}^{*}(t-\tau)\right) \in$ $E^{\perp}$ such that

$$
K\left(v^{*}\right)+H^{\prime *}=X_{v^{*}}^{*}
$$

Hence, $\left\{v^{*}, X_{v^{*}}^{*}\right\}$ is a solution to (4.16). That is, $\left\{\left(v^{*}(t), v^{*}(t-\tau)\right),\left(X_{v^{*}}^{*}(t), X_{v^{*}}^{*}(t-\tau)\right)\right\}$ is a solution to (4.16).

Lemma 5.2. The following two conditions are equivalent:

(1) $F\left(t, u_{1}, u_{2}\right) \leq \alpha_{2}\left[F_{1}^{\prime}\left(t, u_{1}, u_{2}\right) u_{1}+F_{2}^{\prime}\left(t, u_{1}, u_{2}\right) u_{2}\right]$, for all $t \in[0,2 \gamma \tau]$, when $|u|=$ $\sqrt{u_{1}^{2}+u_{2}^{2}} \geq C$

(2) $F\left(t, \beta u_{1}, \beta u_{2}\right) \geq \beta^{1 / \alpha_{2}} F\left(t, u_{1}, u_{2}\right)>0$, for all $\beta \geq 1, t \in[0,2 \gamma \tau],|u| \geq C$.

Proof. For all $u=\left(u_{1}, u_{2}\right),|u| \geq C$, let $\Phi(\beta)=F\left(t, \beta u_{1}, \beta u_{2}\right), \Psi(\beta)=\beta^{1 / \alpha_{2}} F\left(t, u_{1}, u_{2}\right)$.

$(2) \Rightarrow(1)$. By $\Phi(\beta) \geq \Psi(\beta)$, for all $\beta \geq 1$ and $\Phi(1)=\Psi(1)$, it is easy to see that $\Phi^{\prime}(1) \geq$ $\Psi^{\prime}(1)$. That is,

$$
F_{1}^{\prime}\left(t, u_{1}, u_{2}\right) u_{1}+F_{2}^{\prime}\left(t, u_{1}, u_{2}\right) u_{2} \geq \frac{1}{\alpha_{2}} F\left(t, u_{1}, u_{2}\right)
$$


$(1) \Rightarrow(2)$. By

$$
\begin{aligned}
\Phi^{\prime}(\beta) & =F_{1}^{\prime}\left(t, \beta u_{1}, \beta u_{2}\right) u_{1}+F_{2}^{\prime}\left(t, \beta u_{1}, \beta u_{2}\right) u_{2} \\
& =\frac{1}{\beta}\left[F_{1}^{\prime}\left(t, \beta u_{1}, \beta u_{2}\right) \beta u_{1}+F_{2}^{\prime}\left(t, \beta u_{1}, \beta u_{2}\right) \beta u_{2}\right] \geq \frac{1}{\alpha_{2} \beta} \Phi(\beta),
\end{aligned}
$$

it follows that

$$
F\left(t, \beta u_{1}, \beta u_{2}\right) \geq \beta^{1 / \alpha_{2}} F\left(t, u_{1}, u_{2}\right)>0, \quad \forall \beta \geq 1, t \in[0,2 \gamma \tau]
$$

Lemma 5.3. Let $F\left(t, u_{1}, u_{2}\right)$ satisfy assumptions $\left(A_{4}\right)$ and $\left(A_{5}\right)$. Then there exist constants $m>0$ and $M>0$ such that

$$
\begin{gathered}
F\left(t, u_{1}, u_{2}\right) \geq m\left(\sqrt{u_{1}^{2}+u_{2}^{2}}\right)^{1 / \alpha_{2}} \quad \forall t \in[0,2 \gamma \tau], \text { when }|u| \geq C, \\
\left|F^{\prime}\left(t, u_{1}, u_{2}\right)\right| \leq\left(2^{1 / \alpha_{2}} M-m\right)\left(\sqrt{u_{1}^{2}+u_{2}^{2}}\right)^{\left(1 / \alpha_{2}\right)-1}, \quad \text { when }|u| \geq C,
\end{gathered}
$$

where $\left|F^{\prime}\left(t, u_{1}, u_{2}\right)\right|=\sqrt{\left|F_{1}^{\prime}\left(t, u_{1}, u_{2}\right)\right|^{2}+\left|F_{2}^{\prime}\left(t, u_{1}, u_{2}\right)\right|^{2}}$.

Proof. Let

$$
m=\min _{\left(u_{1}, u_{2}\right) \in \partial B_{C}} \frac{F\left(t, u_{1}, u_{2}\right)}{C^{1 / \alpha_{2}}}
$$

where $B_{C}$ denotes the ball of radius $C$ centered at the origin. By $\left(A_{4}\right)$, one knows $m>0$. On the other hand, by Lemma 5.2 and $\left(A_{5}\right)$, we have

$$
\begin{aligned}
F\left(t, u_{1}, u_{2}\right) & \geq F\left(t, \frac{C u_{1}}{\sqrt{u_{1}^{2}+u_{2}^{2}}}, \frac{C u_{2}}{\sqrt{u_{1}^{2}+u_{2}^{2}}}\right)\left(\frac{\sqrt{u_{1}^{2}+u_{2}^{2}}}{C}\right)^{1 / \alpha_{2}} \\
& \geq m\left(\sqrt{u_{1}^{2}+u_{2}^{2}}\right)^{1 / \alpha_{2}} .
\end{aligned}
$$

By the convexity of function $F$, one has

$$
F\left(t, u_{1}, u_{2}\right)+F_{1}^{\prime}\left(t, u_{1}, u_{2}\right)\left(z_{1}-u_{1}\right)+F_{2}^{\prime}\left(t, u_{1}, u_{2}\right)\left(z_{2}-u_{2}\right) \leq F\left(t, z_{1}, z_{2}\right) .
$$


Let $z=\left(z_{1}, z_{2}\right)$ run all over the ball $B_{|u|}(z)$ of radius $|u|$ centered at $u=\left(u_{1}, u_{2}\right)$, and choose the maximum of $F_{1}^{\prime}\left(t, u_{1}, u_{2}\right)\left(z_{1}-u_{1}\right)+F_{2}^{\prime}\left(t, u_{1}, u_{2}\right)\left(z_{2}-u_{2}\right)$. Then it is not difficult to see that

$$
\left|F^{\prime}\left(t, u_{1}, u_{2}\right)\right| \sqrt{u_{1}^{2}+u_{2}^{2}} \leq M\left(\sqrt{z_{1}^{2}+z_{2}^{2}}\right)^{1 / \alpha_{2}}-m\left(\sqrt{u_{1}^{2}+u_{2}^{2}}\right)^{1 / \alpha_{2}} .
$$

By $z \leq 2|u|$ we have that

$$
\left|F^{\prime}\left(t, u_{1}, u_{2}\right)\right| \leq\left(2^{1 / \alpha_{2}} M-m\right)\left(\sqrt{u_{1}^{2}+u_{2}^{2}}\right)^{\left(1 / \alpha_{2}\right)-1} .
$$

Lemma 5.4. $H \in C^{1}\left(\mathbb{R}^{3}, \mathbb{R}\right)$ is a strictly convex function and satisfies

(1)

$$
H_{1}^{\prime}(t, 0,0)=H_{2}^{\prime}(t, 0,0)=0, \quad H(t, 0,0)=0, \quad \forall t \in[0,2 \gamma \tau]
$$

(2)

$$
\frac{C_{\alpha_{2}}}{M}|\omega|^{1 /\left(1-\alpha_{2}\right)}-C_{1} \leq H(t, \omega(t), \omega(t-\tau)) \leq \frac{C_{\alpha_{2}}}{m}|\omega|^{1 /\left(1-\alpha_{2}\right)}+C_{2}
$$

(3)

$$
\begin{aligned}
C_{\alpha_{2}}^{\prime}|\omega|^{\alpha_{2} /\left(1-\alpha_{2}\right)}-C_{4} \leq & \left|H^{\prime}(t, \omega(t), \omega(t-\tau))\right| \leq C_{\alpha_{2}}\left(\frac{2^{1 /\left(1-\alpha_{2}\right)}}{m}-\frac{1}{M}\right) \\
& \times|\omega|^{\alpha_{2} /\left(1-\alpha_{2}\right)}+C_{3} ;
\end{aligned}
$$

$(4)$

$$
\lim _{|\omega| \rightarrow 0} \frac{H(t, \omega(t), \omega(t-\tau))}{|\omega|^{2}}=\infty
$$

where $C_{1}, \ldots, C_{4}$ are constants, $C_{\alpha_{2}}$ and $C_{\alpha_{2}}^{\prime}$ are constants, depending on $\alpha_{2}, \quad|\omega|=\sqrt{\omega^{2}(t)+\omega^{2}(t-\tau)}$ and $\left|H^{\prime}(t, \omega(t), \omega(t-\tau))\right|=$ $\sqrt{\left|H_{1}^{\prime}(t, \omega(t), \omega(t-\tau))\right|^{2}+\left|H_{2}^{\prime}(t, \omega(t), \omega(t-\tau))\right|^{2}}$.

Proof. By Corollary 3.8 and $F_{1}^{\prime}(t, 0,0)=F_{2}^{\prime}(t, 0,0)=0$, we have $H_{1}^{\prime}(t, 0,0)=H_{2}^{\prime}(t, 0,0)=$ 0 , for all $t \in[0,2 \gamma \tau]$. Moreover, by the definition of $H$, we know that $H(t, 0,0)=0$. 
Now we show that $(5.17)$ holds. By $\left(A_{5}\right)$ one obtains $F\left(t, u_{1}, u_{2}\right) \leq M|u|^{1 / \alpha_{2}}+$ $C_{1}$, for all $u=\left(u_{1}, u_{2}\right) \in \mathbb{R}^{2}$. So, by Proposition 3.3 and Example 4.2 , it is easy to see that

$$
H(t, \omega(t), \omega(t-\tau)) \geq \frac{C_{\alpha_{2}}}{M}|\omega|^{1 /\left(1-\alpha_{2}\right)}-C_{1}
$$

where $C_{\alpha_{2}}=2^{1 /\left(1-\alpha_{2}\right)} / M^{\alpha_{2} /\left(1-\alpha_{2}\right)-1}\left(\alpha_{2}^{\alpha_{2} /\left(1-\alpha_{2}\right)}-\alpha_{2}^{1 /\left(1-\alpha_{2}\right)}\right)$.

Similar arguments to the proof of Lemma 5.3 show that there exists a constant $C_{2}$ such that

$$
F\left(t, u_{1}, u_{2}\right) \geq m|u|^{1 / \alpha_{2}}-C_{2}
$$

Therefore, it follows that

$$
H(t, \omega(t), \omega(t-\tau)) \leq \frac{C_{\alpha_{2}}}{m}|\omega|^{1 /\left(1-\alpha_{2}\right)}+C_{2} .
$$

Next we show that (5.18) holds. Again as in the proof of Lemma 5.3, we can estimate $H^{\prime}$ by

$$
\left|H^{\prime}(t, \omega(t), \omega(t-\tau))\right| \leq C_{\alpha_{2}}\left(\frac{2^{1 /\left(1-\alpha_{2}\right)}}{m}-\frac{1}{M}\right)|\omega|^{\alpha_{2} /\left(1-\alpha_{2}\right)}+C_{3}
$$

where $C_{3}=\max \left\{C_{1}+C_{2}, \sup _{|\omega|<1}\left|H^{\prime}(t, \omega(t), \omega(t-\tau))\right| \mid\right\}$. By Lemma 5.3 again and the duality principle

$$
\begin{aligned}
\left(u_{1}, u_{2}\right) & =\left(H_{1}^{\prime}(t, \omega(t), \omega(t-\tau)), H_{2}^{\prime}(t, \omega(t), \omega(t-\tau))\right) \\
& \Longleftrightarrow(\omega(t), \omega(t-\tau))=\left(F_{1}^{\prime}\left(t, u_{1}, u_{2}\right), F_{2}^{\prime}\left(t, u_{1}, u_{2}\right)\right)
\end{aligned}
$$

when $\left|H^{\prime}(t, \omega(t), \omega(t-\tau))\right| \geq C$, we have

$$
|\omega| \leq\left(2^{1 / \alpha_{2}} M-m\right) \mid H^{\prime\left(1 / \alpha_{2}\right)-1}
$$

Since there exists a constant $M_{C}$ such that when $|u|=\sqrt{u_{1}^{2}+u_{2}^{2}}=\left|H^{\prime}(t, \omega(t), \omega(t-\tau))\right| \leq C$, we have

$$
|\omega|=\left|F^{\prime}\left(t, u_{1}, u_{2}\right)\right| \leq M_{C}
$$

Choose

$$
C_{\alpha_{2}}^{\prime}=\left(2^{1 / \alpha_{2}} M-m\right)^{\alpha_{2} /\left(\alpha_{2}-1\right)}, \quad C_{4}=C_{\alpha_{2}}^{\prime} M_{C}^{\alpha_{2} /\left(1-\alpha_{2}\right)} .
$$

Then it is not difficult to see

$$
\left|H^{\prime}(t, \omega(t), \omega(t-\tau))\right| \geq C_{\alpha_{2}}^{\prime}|\omega|^{\alpha_{2} /\left(1-\alpha_{2}\right)}-C_{4} .
$$


Finally, we show that (5.19) holds. By $\left(A_{6}\right)$, for all $\varepsilon>0$, there exists $\delta>0$ so that when $|u|=\sqrt{u_{1}^{2}+u_{2}^{2}}<\delta$, we have

$$
F\left(t, u_{1}, u_{2}\right) \leq \varepsilon \sqrt{u_{1}^{2}+u_{2}^{2}}
$$

Now, for all $K>0$, choose $\varepsilon=1 / 4 K$ and let $\eta=2 \varepsilon \delta(\varepsilon)$. Then whenever $\sqrt{\omega^{2}(t)+\omega^{2}(t-\tau)}<$ $\eta$, we have

$$
H(t, \omega(t), \omega(t-\tau)) \geq \frac{1}{4 \varepsilon}\left(\omega^{2}(t)+\omega^{2}(t-\tau)\right)=K|\omega|^{2}
$$

That is,

$$
\lim _{|\omega| \rightarrow 0} \frac{H(t, \omega(t), \omega(t-\tau))}{|\omega|^{2}}=\infty
$$

Lemma 5.5. There exist constants $C_{\delta}$ and $C_{\delta}^{\prime}$ depending on $\delta$, such that

$$
H(t, \omega(t), \omega(t-\tau)) \geq \begin{cases}C_{\delta}|\omega|^{2}, & \text { when }|\omega| \leq \delta \\ C_{\delta}^{\prime}|\omega|^{q}, & \text { when }|\omega|>\delta\end{cases}
$$

and when $\delta \rightarrow+0, C_{\delta} \rightarrow+\infty$.

Proof. By (5.19), we have

$$
\lim _{|\omega| \rightarrow 0} \frac{H(t, \omega(t), \omega(t-\tau))}{|\omega|^{2}}=\infty
$$

So, as $\delta \rightarrow+0$, we obtain $C_{\delta} \triangleq \inf \left\{H(t, \omega(t), \omega(t-\tau)) /|\omega|^{2}:|\omega| \leq \delta\right\} \rightarrow+\infty$. That is,

$$
H(t, \omega(t), \omega(t-\tau)) \geq C_{\delta}|\omega|^{2}
$$

when $|\omega| \leq \delta$.

We next show the validity of the second part of the inequality.

For all $\omega_{0}=\left(\omega_{0}(t), \omega_{0}(t-\tau)\right),\left|\omega_{0}\right|=1$, let $\phi_{\omega_{0}}(\beta)=H\left(t, \beta \omega_{0}(t), \beta \omega_{0}(t-\tau)\right)$. Then

$$
\phi_{\omega_{0}}^{\prime}(\beta)=H_{1}^{\prime}\left(t, \beta \omega_{0}(t), \beta \omega_{0}(t-\tau)\right) \omega_{0}(t)+H_{2}^{\prime}\left(t, \beta \omega_{0}(t), \beta \omega_{0}(t-\tau)\right) \omega_{0}(t-\tau)
$$

Since $\phi_{\omega_{0}}$ is a convex function for all $\beta>0$, we have

$$
H_{1}^{\prime}\left(t, \beta \omega_{0}(t), \beta \omega_{0}(t-\tau)\right) \omega_{0}(t)+H_{2}^{\prime}\left(t, \beta \omega_{0}(t), \beta \omega_{0}(t-\tau)\right) \omega_{0}(t-\tau) \geq \frac{1}{\beta} \phi_{\omega_{0}}(\beta)
$$


So, by (5.34) we obtain

$$
H_{1}^{\prime}\left(t, \delta \omega_{0}(t), \delta \omega_{0}(t-\tau)\right) \omega_{0}(t)+H_{2}^{\prime}\left(t, \delta \omega_{0}(t), \delta \omega_{0}(t-\tau)\right) \omega_{0}(t-\tau) \geq C_{\delta} \cdot \delta
$$

By the convexity of $H$ again, it is easy to see that

$$
\begin{aligned}
H\left(t, s \omega_{0}(t), s \omega_{0}(t-\tau)\right) \geq & H_{1}^{\prime}\left(t, \delta \omega_{0}(t), \delta \omega_{0}(t-\tau)\right)(s-\delta) \omega_{0}(t) \\
& +H_{2}^{\prime}\left(t, \delta \omega_{0}(t), \delta \omega_{0}(t-\tau)\right)(s-\delta) \omega_{0}(t-\tau) \\
& +H\left(t, \delta \omega_{0}(t), \delta \omega_{0}(t-\tau)\right) \\
\geq & C_{\delta} \cdot \delta(s-\delta)+C_{\delta} \delta^{2}=C_{\delta} \delta s, \quad \forall s>0 .
\end{aligned}
$$

So, we further obtain that

$$
H(t, \omega(t), \omega(t-\tau)) \geq C_{\delta} \cdot \delta|\omega|
$$

By Lemma 5.4, there exists $T>0$ such that

$$
H(t, \omega(t), \omega(t-\tau)) \geq \frac{C_{\alpha_{2}}}{2 M}|\omega|^{q}
$$

Let $C_{\delta}^{\prime}=\min \left\{C_{\alpha_{2}} / 2 M, T^{1-q} \delta C_{\delta}\right\}$. Then by (5.34), (5.39), and (5.40), we have

$$
H(t, \omega(t), \omega(t-\tau)) \geq \begin{cases}C_{\delta}|\omega|^{2}, & |\omega| \leq \delta \\ C_{\delta}^{\prime}|\omega|^{q}, & |\omega|>\delta\end{cases}
$$

Lemma 5.6. Let $v_{m}=\left(v_{m}(t), v_{m}(t-\tau)\right) \rightarrow v=(v(t), v(t-\tau))$ (weakly convergent sequence on $\left.L^{q}([0,2 \gamma \tau])^{2}\right)$ and satisfy

$$
\int_{0}^{2 \gamma \tau} H\left(t, v_{m}\right) d t \longrightarrow \int_{0}^{2 \gamma \tau} H(t, v) d t \quad \text { as } m \longrightarrow \infty
$$

Then

$$
\int_{0}^{2 \gamma \tau} H\left(t, v_{m}-v\right) d t \longrightarrow 0 \quad \text { as } m \longrightarrow \infty
$$

Proof. (I) First, we show that the terms in $\left\{H\left(t, v_{m}\right)\right\}$ have equicontinuous integrals, that is, for all $\varepsilon>0$, there exists $\delta>0$ such that

$$
\forall m, \int_{\Omega} H\left(t, v_{m}\right) d t<\varepsilon \quad \text { when } \mu(\Omega)<\delta
$$


Since $H$ is convex, we have

$$
H_{1}^{\prime}(t, v)\left(v(t)-v_{m}(t)\right)+H_{2}^{\prime}(t, v)\left(v(t-\tau)-v_{m}(t-\tau)\right) \leq H\left(t, v_{m}\right)-H(t, v)
$$

So, from $v_{m} \rightarrow v$ and the above equality, we have

$$
\int_{0}^{2 \gamma \tau} H(t, v) d t \leq \lim _{m \rightarrow \infty} \int_{0}^{2 \gamma \tau} H\left(t, v_{m}\right) d t
$$

By $H \geq 0$ and the assumption that $\int_{0}^{2 \gamma \tau} H(t, v) d t \rightarrow \int_{0}^{2 \gamma \tau} H\left(t, v_{m}\right) d t$, it is not difficult to see

$$
\lim _{m \rightarrow \infty} \int_{\Omega} H\left(t, v_{m}\right) d t=\int_{\Omega} H(t, v) d t \quad \text { for all measurable sets } \Omega
$$

Now suppose to the contrary that $\left\{H\left(t, v_{m}\right)\right\}$ does not have equicontinuous integrals, that is, there exists $\varepsilon_{0}>0$ and functions $v_{m_{k}}=\left(v_{m_{k}}(t), v_{m_{k}}(t-\tau)\right)$, as well as measurable sets $\Omega_{k}$, such that the following inequalities

$$
\begin{gathered}
\int_{\Omega} H(t, \pm v) d t<\varepsilon_{0}, \quad \text { for all measurable sets } \Omega, \mu(\Omega)<\delta, \\
\int_{\Omega_{k}} H\left(t, v_{m_{k}}\right) d t \geq \varepsilon_{0}, \quad \mu\left(\Omega_{k}\right)<\frac{\delta}{2^{k}}
\end{gathered}
$$

hold. Then choose $\Omega_{0}=\bigcup_{k=1}^{\infty} \Omega_{k}$. It is not difficult to obtain $\mu\left(\Omega_{0}\right)<\delta$ and

$$
\int_{\Omega_{0}} H\left(t, v_{m_{k}}\right) d t \geq \int_{\Omega_{k}} H\left(t, v_{m_{k}}\right) d t \geq \varepsilon_{0}
$$

which is contradictory to (5.46) and (5.47).

(II) For all $b>0$, we divide $[0,2 \gamma \tau]$ into the following three subsets:

$$
\begin{gathered}
Q_{1}=\left\{t \in[0,2 \gamma \tau]|| v \mid=\sqrt{v^{2}(t)+v^{2}(t-\tau)}>b\right\}, \\
Q_{2}^{m}=\left\{t \in[0,2 \gamma \tau]|| v|\leq b,| v_{m}-v \mid \geq \delta\right\}, \\
Q_{3}^{m}=\left\{t \in[0,2 \gamma \tau]|| v|\leq b,| v_{m}-v \mid<\delta\right\},
\end{gathered}
$$

where $\left|v_{m}-v\right|=\sqrt{\left(v_{m}(t)-v(t)\right)^{2}+\left(v_{m}(t-\tau)-v(t-\tau)\right)^{2}}$. By inequality (5.17), we know that there exist constants $K$ and $L$ such that

$$
H(t, 2 z(t), 2 z(t-\tau)) \leq K H(t, z(t), z(t-\tau))+L, \quad \forall(z(t), z(t-\tau)) \in \mathbb{R}^{2} .
$$


By the convexity of the function $H$ on $Q_{1}$, we obtain

$$
H\left(t, v_{m}-v\right) \leq \frac{1}{2}\left[H\left(t, 2 v_{m}\right)+H(t,-2 v)\right] \leq \frac{K}{2}\left(H\left(t, v_{m}\right)+H(t,-v)\right)+L
$$

So by (I), we may choose a constant $b$ large enough and fixed such that $\mu\left(Q_{1}\right)$ is small enough so that

$$
\int_{Q_{1}} H\left(t, v_{m}-v\right) d t \leq \frac{K}{2} \int_{Q_{1}}\left(H\left(t, v_{m}\right)+H(t,-v)\right) d t+L \mu\left(Q_{1}\right)<\frac{\varepsilon}{3}
$$

For the above chosen constant $b$, now choose $\delta$ small enough so that

$$
\int_{Q_{3}^{m}} H\left(t, v_{m}-v\right) d t<\frac{\varepsilon}{3}
$$

For the chosen constants $b$ and $\delta$, let

$$
\kappa=\inf _{|\omega-z| \geq \delta,|z| \leq b}\left[H(t, \omega)-H(t, z)-H_{1}^{\prime}(t, \omega)(\omega(t)-z(t))-H_{2}^{\prime}(t, \omega)(\omega(t-\tau)-z(t-\tau))\right]
$$

Then $\kappa>0$. Now

$$
\begin{aligned}
\kappa \mu\left(Q_{2}^{m}\right) & \leq \int_{Q_{2}^{m}}\left[H\left(t, v_{m}\right)-H(t, v)-H_{1}^{\prime}(t, v)\left(v_{m}(t)-v(t)\right)-H_{2}^{\prime}(t, v)\left(v_{m}(t-\tau)-v(t-\tau)\right)\right] d t \\
& \leq \int_{0}^{2 \gamma \tau}\left[H\left(t, v_{m}\right)-H(t, v)-H_{1}^{\prime}(t, v)\left(v_{m}(t)-v(t)\right)-H_{2}^{\prime}(t, v)\left(v_{m}(t-\tau)-v(t-\tau)\right)\right] d t .
\end{aligned}
$$

Thus we have $\mu\left(Q_{2}^{m}\right) \rightarrow 0$ as $m \rightarrow \infty$. Hence, it is easy to see that $\int_{Q_{2}^{m}} H\left(t, v_{m}\right) \rightarrow 0$ as $m \rightarrow \infty$.

By repeating the above argument on $Q_{1}$, we conclude that there exists an $n_{0}$ such that when $m>n_{0}$, we have

$$
\int_{Q_{2}^{m}} H\left(t, v_{m}-v\right) d t<\frac{\varepsilon}{3} .
$$

From (5.53), (5.54), and (5.57), we obtain that

$$
\lim _{m \rightarrow \infty} \int_{0}^{2 \gamma \tau} H\left(t, v_{m}-v\right) d t=0
$$


Corollary 5.7. $v_{m}=\left(v_{m}(t), v_{m}(t-\tau)\right) \rightarrow v=(v(t), v(t-\tau))\left(L^{q}([0,2 \gamma \tau]) \times L^{q}([0,2 \gamma \tau])\right)$ if and only if

$$
\int_{0}^{2 \gamma \tau} H\left(t, v_{m}-v\right) d t=0
$$

Proof. $(\Rightarrow) . v_{m} \rightarrow v$ implies that $v_{m} \rightarrow v$ (weakly). By inequality (5.17) and the continuity of the composition operator, one gets $H\left(t, v_{m}\right) \rightarrow H(t, v)\left(L^{1}([0,2 \gamma \tau])\right)$. That is, $\int_{0}^{2 \gamma \tau} H\left(t, v_{m}\right) d t \rightarrow \int_{0}^{2 \gamma \tau} H(t, v) d t$. So, by Lemma 5.6, one obtains the conclusion.

$(\Leftarrow)$. By Lemma 5.5, there exist constants $B_{1}$ and $B_{2}>0$ such that

$$
\begin{aligned}
\int_{0}^{2 \gamma \tau} H(t, v) d t & \geq B_{1} \int_{|v| \geq \delta}|v|^{q} d t+B_{2} \int_{|v|<\delta}|v|^{2} d t \\
& \geq B_{1} \int_{|v| \geq \delta}|v|^{q} d t+B_{2}(2 \gamma \tau)^{2 /(q-2)}\left(\int_{|v|<\delta}|v|^{q} d t\right)^{2 / q} \\
& \geq C_{\delta} \min \left\{\int_{0}^{2 \gamma \tau}|v|^{q} d t,\left(\int_{0}^{2 \gamma \tau}|v|^{q} d t\right)^{2 / q}\right\}
\end{aligned}
$$

Choose $\delta$ small enough. Then $C_{\delta}>0$ is a constant and it is not difficult to see that the conclusion is correct.

We next use the Maintain Pass Theorem to prove Theorem 5.1 in three steps.

(i) We show that $J$ satisfies the P. S. condition in $E$. Let $\left\{v_{n}=\left(v_{n}(t), v_{n}(t-\tau)\right)\right\} \subset E$, and let the constants $C_{1}, C_{2}$ satisfy

$$
\begin{gathered}
C_{1} \leq J\left(v_{n}\right) \leq C_{2}, \\
J^{\prime}\left(v_{n}\right) \longrightarrow \theta .
\end{gathered}
$$

That is, we want to show that $\left\{v_{n}\right\}$ has a convergent subsequence in $E$.

First, we show that $\left\{v_{n}\right\}$ is bounded. In fact, by

$$
\begin{gathered}
z_{m}=K v_{m}+H^{\prime}\left(t, v_{m}\right)-x_{m} \longrightarrow \theta \\
C_{1} \leq \frac{1}{2}\left\langle K v_{m}, v_{m}\right\rangle+\int_{0}^{2 \gamma \tau} H\left(t, v_{m}\right) d t \leq C_{2},
\end{gathered}
$$


where $z_{m}=\left(z_{m}(t), z_{m}(t-\tau)\right), v_{m}=\left(v_{m}(t), v_{m}(t-\tau)\right), \chi_{m}=\left(\chi_{m}(t), \chi_{m}(t-\tau)\right)$, we know that there exists $n(\varepsilon)>0$ for all $\varepsilon>0$ such that the following inequality holds when $m \geq m(\varepsilon)$ :

$$
\begin{aligned}
& \int_{0}^{2 \gamma \tau} H\left(t, v_{m}\right) d t-\frac{1}{2}\left[H_{1}^{\prime}\left(t, v_{m}\right) v_{m}(t)+H_{2}^{\prime}\left(t, v_{m}\right) v_{m}(t-\tau)\right] \\
& \quad \leq C_{2}+\frac{\varepsilon}{2}\left(\left\|v_{m}(t)\right\|_{L^{q}}+\left\|v_{m}(t-\tau)\right\|_{L^{q}}\right)=C_{2}+\varepsilon\left\|v_{m}(t)\right\|_{L^{q}}
\end{aligned}
$$

On the other hand, by Lemmas 5.2 and 5.3, there exist constants $\alpha_{2}, C_{3}, C_{4}$ and $C_{5}$ such that

$$
\begin{aligned}
H(t, & \omega)-\frac{1}{2} H_{1}^{\prime}(t, \omega) \omega(t)-\frac{1}{2} H_{2}^{\prime}(t, \omega) \omega(t-\tau) \\
= & \frac{1}{2} z(t) F_{1}^{\prime}(t, z(t), z(t-\tau))+\frac{1}{2} z(t-\tau) F_{2}^{\prime}(t, z(t), z(t-\tau))-F(t, z(t), z(t-\tau)) \\
\geq & \left(\frac{1}{2 \alpha_{2}}-1\right) F(t, z(t), z(t-\tau))-C_{3} \geq m|z|^{1 / \alpha_{2}}\left(\frac{1}{2 \alpha_{2}}-1\right)-C_{4} \\
& \geq|\omega|^{q}-C_{5}
\end{aligned}
$$

where $\omega(t)=F_{1}^{\prime}(t, z(t), z(t-\tau)), \omega(t-\tau)=F_{2}^{\prime}(t, z(t), z(t-\tau)) ; z(t)=H_{1}^{\prime}(t, \omega(t), \omega(t-\tau))$, $z(t-\tau)=H_{2}^{\prime}(t, \omega(t), \omega(t-\tau)) ;|\omega|=\sqrt{\omega^{2}(t)+\omega^{2}(t-\tau)} ;|z|=\sqrt{z^{2}(t)+z^{2}(t-\tau)}$.

So by (5.63) and (5.64), it is easy to see that

$$
\left\|v_{m}(t)\right\|_{L^{q}[0,2 \gamma \tau]}=\left\|v_{m}(t-\tau)\right\|_{L^{q}[0,2 \gamma \tau]} \leq C_{6} \quad(\text { constant })
$$

That is, $\left\{v_{n}\right\}$ is bounded. We next show that $\left\{v_{n}\right\}$ has a convergent subsequence. Since $L^{q}[0,2 \gamma \tau]$ is a reflexive Banach space, there exists a subsequence of $\left\{v_{n}\right\}$ which is weakly convergent in $L^{q}[0,2 \gamma \tau]$. We denote it by $\left\{v_{m_{k}}\right\}$, that is to say, $v_{m_{k}}(t) \rightarrow v^{*}(t), v_{m_{k}}(t-\tau) \rightarrow$ $v^{*}(t-\tau)$. On one hand, by the convexity of the function $H$, we have

$$
\begin{aligned}
& H\left(t, v^{*}(t), v^{*}(t-\tau)\right)+H_{1}^{\prime}\left(t, v^{*}(t), v^{*}(t-\tau)\right)\left(v_{m_{k}}(t)-v^{*}(t)\right) \\
& \quad+H_{2}^{\prime}\left(t, v^{*}(t), v^{*}(t-\tau)\right)\left(v_{m_{k}}(t-\tau)-v^{*}(t-\tau)\right) \leq H\left(t, v_{m_{k}}(t), v_{m_{k}}(t-\tau)\right) .
\end{aligned}
$$

So we have

$$
\int_{0}^{2 \gamma \tau} H\left(t, v^{*}(t), v^{*}(t-\tau)\right) d t \leq \lim _{k \rightarrow \infty} \int_{0}^{2 \gamma \tau} H\left(t, v_{m_{k}}(t), v_{m_{k}}(t-\tau)\right) d t
$$


On the other hand, by convexity of the function $H$ again, we obtain

$$
\begin{aligned}
H\left(t, v^{*}(t), v^{*}(t-\tau)\right) \geq & H\left(t, v_{m_{k}}(t), v_{m_{k}}(t-\tau)\right)+H_{1}^{\prime}\left(t, v_{m_{k}}(t), v_{m_{k}}(t-\tau)\right)\left(v^{*}(t)-v_{m_{k}}(t)\right) \\
& +H_{2}^{\prime}\left(t, v_{m_{k}}(t), v_{m_{k}}(t-\tau)\right)\left(v^{*}(t-\tau)-v_{m_{k}}(t-\tau)\right) \\
= & H\left(t, v_{m_{k}}(t), v_{m_{k}}(t-\tau)\right)+\left(-K v_{m_{k}}+z_{m_{k}}+X_{m_{k}}\right) \cdot\left(v^{*}-v_{m_{k}}\right) .
\end{aligned}
$$

Since operators $A$ and $K$ are compact and $\left(z_{m_{k}}(t), z_{m_{k}}(t-\tau)\right) \rightarrow \theta$, we have

$$
\varlimsup_{k \rightarrow \infty} \int_{0}^{2 \gamma \tau} H\left(t, v_{m_{k}}(t), v_{m_{k}}(t-\tau)\right) d t \leq \int_{0}^{2 \gamma \tau} H\left(t, v^{*}(t), v^{*}(t-\tau)\right) d t .
$$

By (5.67), (5.69), and applying Lemma 5.6 and Corollary 5.7, it is not difficult to see that

$$
\left(v_{m_{k}}(t), v_{m_{k}}(t-2 \tau)\right) \longrightarrow\left(v^{*}(t), v^{*}(t-\tau)\right) .
$$

(ii) We show that there exist constants $\rho, r>0$, such that

$$
\left.J\right|_{\partial \Omega_{r}} \geq \rho>0
$$

where $\partial \Omega_{r}=\left\{(v(t), v(t-\tau)) \in L^{q}[0,2 \gamma \tau] \times L^{q}[0,2 \gamma \tau] \mid\|v(t)\|_{L^{q}[0,2 \gamma \tau]}=\|v(t-\tau)\|_{L^{q}[0,2 \gamma \tau]}=r\right\}$. Let $\beta=\|\widehat{K}\|_{E\left(L^{p}, L^{q}\right)}$. Choose $\delta>0$ such that the constant $C_{\delta}$ is large enough and choose $r$ small enough so that when $\|v(t)\|_{L^{q}}=r$, by Lemma 5.5, there exists constant $C_{7}>0$ satisfying

$$
\begin{aligned}
& C_{\delta} \int_{|v|<\delta}|v(t)|^{2} d t-4 \beta\left(\int_{|v|<\delta}|v(t)|^{q} d t\right)^{2 / q} \geq C_{7}\left(\int_{|v|<\delta}|v(t)|^{q} d t\right)^{2 / q}, \\
& C_{\delta}^{\prime} \int_{|v| \geq \delta}|v(t)|^{q} d t-4 \beta\left(\int_{|v| \geq \delta}|v(t)|^{q} d t\right)^{2 / q} \geq C_{7}\left(\int_{|v| \geq \delta}|v(t)|^{q} d t\right)^{2 / q},
\end{aligned}
$$

where $|v|=\sqrt{v^{2}(t)+v^{2}(t-\tau)}$. 
Abstract and Applied Analysis

By (5.72) and inequalities

$$
a^{c}+b^{c} \leq(a+b)^{c} \leq 2^{c}\left(a^{c}+b^{c}\right),
$$

where $a, b>0$, and $c>1$, we have

$$
\begin{aligned}
J(v)= & \frac{1}{2}\langle\widehat{K}(v(t)), v(t-\tau)\rangle+\frac{1}{2}\langle\widehat{K}(v(t-\tau)), v(t)\rangle+\int_{0}^{2 \gamma \tau} H(t, v(t), v(t-\tau)) \\
\geq & -\frac{\beta}{2}\|v(t)\|_{L^{q}[0,2 \gamma \tau]}^{2}-\frac{\beta}{2}\|v(t)\|_{L^{q}[0,2 \gamma \tau]}^{2} \\
& +C_{\delta} \int_{|v|<\delta}|v(t)|^{2} d t+C_{\delta} \int_{|v|<\delta}|v(t-\tau)|^{2} d t+C_{\delta}^{\prime} \int_{|v| \geq \delta}\left(\sqrt{v^{2}(t)+v^{2}(t-\tau)}\right)^{q} d t \\
\geq & -\frac{\beta}{2}\|v(t)\|_{L^{q}[0,2 \gamma \tau]}^{2}-\frac{\beta}{2}\|v(t)\|_{L^{q}[0,2 \gamma \tau]}^{2}+C_{\delta} \int_{|v|<\delta}|v(t)|^{2} d t \\
& +C_{\delta} \int_{|v|<\delta}|v(t-\tau)|^{2} d t+C_{\delta}^{\prime} \int_{|v| \geq \delta}|v(t)|^{q} d t \\
\geq & C_{7}\left[\left(\int_{|v|<\delta}|v(t)|^{q} d t\right)^{2 / q}+\left(\int_{|v| \geq \delta}|v(t)|^{q} d t\right)^{2 / q}\right]+C_{\delta} \int_{|v|<\delta}|v(t-\tau)|^{2} d t \\
\geq & \frac{C_{7}}{2^{2 / q}}\|v(t)\|_{L^{q}[0,2 \gamma \tau]}^{2}=\frac{C_{7}}{2^{2 / q}} r^{2} .
\end{aligned}
$$

Thus (5.71) is true with $\rho=\left(C_{7} / 2^{2 / q}\right) r^{2}$.

(iii) It is obvious that $J(\theta)=0$, and $J(v)$ is an even function in $v$.

Remark 5.8. Let $v_{j}(t)=\sin (j \pi / \gamma \tau) t, j=1,2, \ldots$ Then by

$$
A v_{j}(t)=-\left(\frac{j \pi}{\gamma \tau}\right)^{2} \sin \frac{j \pi}{\gamma \tau} t
$$

we have

$$
\widehat{K} v_{j}(t)=-\left(\frac{\gamma \tau}{j \pi}\right)^{2} \sin \frac{j \pi}{\gamma \tau} t
$$

Hence, we obtain

$$
\begin{gathered}
\widehat{K} v_{j}(t)=-\left(\frac{\gamma \tau}{j \pi}\right)^{2}\left[\sin \frac{2 j \pi}{\gamma} \cos \frac{j \pi}{\gamma \tau}(t-2 \tau)+\cos \frac{2 j \pi}{\gamma} \sin \frac{j \pi}{\gamma \tau}(t-2 \tau)\right], \\
\widehat{K} v_{j}(t-2 \tau)=-\left(\frac{\gamma \tau}{j \pi}\right)^{2}\left[\cos \frac{2 j \pi}{\gamma} \sin \frac{j \pi}{\gamma \tau} t-\sin \frac{2 j \pi}{\gamma} \cos \frac{j \pi}{\gamma \tau} t\right] .
\end{gathered}
$$


Therefore, it is easy to see

$$
\begin{aligned}
J\left(v_{j}\right) & =\frac{1}{2}\left\langle\widehat{K} v_{j}(t-2 \tau), v_{j}(t)\right\rangle+\frac{1}{2}\left\langle\widehat{K} v_{j}(t), v_{j}(t-2 \tau)\right\rangle+\int_{0}^{2 \gamma \tau} H\left(t, v_{j}\right) d t \\
& =-\frac{1}{2}\left(\frac{\gamma \tau}{j \pi}\right)^{2} \cos \frac{2 j \pi}{\gamma}\left[\int_{0}^{2 \gamma \tau}\left|v_{j}(t)\right|^{2} d t+\int_{0}^{2 \gamma \tau}\left|v_{j}(t-2 \tau)\right|^{2} d t\right]+\int_{0}^{2 \gamma \tau} H\left(t, v_{j}\right) d t .
\end{aligned}
$$

We may choose $j_{k}$, such that $\cos 2 j_{k} \pi / \gamma>0$. Let $v_{j_{k}}(t)=\sin \left(j_{k} \pi / \gamma \tau\right) t$. By (5.78), we have

$$
\begin{aligned}
J\left(\lambda v_{j_{k}}\right) \leq & -\lambda \frac{1}{2}\left(\frac{\gamma \tau}{j \pi}\right)^{2} \cos \frac{2 j \pi}{\gamma}\left[\int_{0}^{2 \gamma \tau}|\phi(t)|^{2} d t+\int_{0}^{2 \gamma \tau}|\phi(t-2 \tau)|^{2} d t\right] \\
& +\frac{C_{\alpha_{2}}}{m} \int_{0}^{2 \gamma \tau}\left(\sqrt{\phi^{2}(t)+\phi^{2}(t-2 \tau)}\right)^{q} d t+2 \gamma \tau C_{2} \longrightarrow-\infty, \quad(\lambda \longrightarrow+\infty) .
\end{aligned}
$$

From (i), (ii), (iii), and the Maintain Pass Theorem, we conclude that problem (4.16) has at least one nontrivial $2 \gamma \tau$-periodic solution. Thus, Theorem 5.1 holds.

\section{Example}

In this section, we present a remark and an example to illustrate our main result.

Remark 6.1. For assumptions $\left(A_{1}\right) \sim\left(A_{3}\right)$, function $F$ is a solution to the following partial differential equation:

$$
F_{2}^{\prime}\left(t, x_{1}, x_{2}\right)+F_{1}^{\prime}\left(t, x_{2}, x_{3}\right)=f\left(t, x_{1}, x_{2}, x_{3}\right)
$$

In some special cases, $F$ can be easily determined. For example, if there exist continuously differential functions $g, h \in C^{1}\left(\mathbb{R}^{2}, \mathbb{R}\right)$ such that $(\partial g / \partial u)(u, v)=(\partial h / \partial v)(u, v)$ and $f\left(t, x_{1}, x_{2}, x_{3}\right)=\alpha(t)\left[g\left(x_{1}, x_{2}\right)+h\left(x_{2}, x_{3}\right)\right]$, where $\alpha(t)$ is continuously differentiable and $\tau$ periodic in $t$, then

$$
F\left(t, x_{1}, x_{2}\right)=\alpha(t)\left[\int_{0}^{x_{1}} h\left(s, x_{2}\right) d s+\int_{0}^{x_{2}} g(0, s) d s\right]
$$

or

$$
F\left(t, x_{1}, x_{2}\right)=\alpha(t)\left[\int_{0}^{x_{2}} g\left(x_{1}, s\right) d s+\int_{0}^{x_{1}} h(s, 0) d s\right]
$$


For example,

$(\mathbb{1})$ if $g(u, v)=u^{2}-2 u v-v^{2}, h(u, v)=u^{2}+2 u v-v^{2}$ and $f\left(t, x_{1}, x_{2}, x_{3}\right)=\cos (2 \tau t / \pi)\left[x_{1}^{2}-\right.$ $\left.2 x_{1} x_{2}+2 x_{2} 2 x_{3}-x_{3}^{2}\right]$, then

$$
\begin{aligned}
F\left(t, x_{1}, x_{2}\right) & =\cos \frac{2 \pi t}{\pi}\left[\int_{0}^{x_{2}} g\left(x_{1}, s\right) d s+\int_{0}^{x_{1}} h(s, 0) d s\right] \\
& =\cos \frac{2 \pi t}{\pi}\left[\int_{0}^{x_{2}}\left(x_{1}^{2}-2 x_{1} s-s^{2}\right) d s+\int_{0}^{x_{1}} s^{2} d s\right] \\
& =\cos \frac{2 \pi t}{\pi}\left(x_{1}^{2} x_{2}-x_{1} x_{2}^{2}-\frac{1}{3} x_{2}^{3}+\frac{1}{3} x_{1}^{3}\right)
\end{aligned}
$$

(2) if $g(u, v)=2 v \cos u-u^{2} \sin v, h(u, v)=2 u \cos v-v^{2} \sin u$ and $f\left(t, x_{1}, x_{2}, x_{3}\right)=$ $\cos (2 \tau t / \pi)\left[2 x_{2} \cos x_{1}-x_{1}^{2} \sin x_{2}+2 x_{2} \cos x_{3}-x_{3}^{2} \sin x_{2}\right]$, then

$$
\begin{aligned}
F\left(t, x_{1}, x_{2}\right) & =\cos \frac{2 \pi t}{\pi}\left[\int_{0}^{x_{2}} g\left(x_{1}, s\right) d s+\int_{0}^{x_{1}} h(s, 0) d s\right] \\
& =\cos \frac{2 \pi t}{\pi}\left[\int_{0}^{x_{2}}\left(2 s \cos x_{1}-x_{1}^{2} \sin s\right) d s+\int_{0}^{x_{1}} 2 s d s\right] \\
& =\cos \frac{2 \pi t}{\pi}\left(x_{2}^{2} \cos x_{1}+x_{1}^{2} \cos x_{2}\right) .
\end{aligned}
$$

One can check easily that $F$ satisfies assumption $\left(A_{2}\right)$.

Finally, as an application, we consider the following example.

Example 6.2. Consider the equation

$$
x^{\prime \prime}(t)+x^{\prime \prime 2}\left(\frac{2 \tau t}{\pi}\right) x(t-\tau)\left[\left(x^{2}(t)+x^{2}(t-\tau)\right)^{(p / 2)-1}+\left(x^{2}(t-\tau)+x^{2}(t-2 \tau)\right)^{(p / 2)-1}\right]=0
$$

with $x(0)=0$, where $p>2$. Then $g(u, v)=p v\left(u^{2}+v^{2}\right)^{(p / 2)-1}$ and $h(u, v)=p u\left(u^{2}+v^{2}\right)^{(p / 2)-1}$. choose

Moreover, $g$ and $h$ satisfy the condition $(\partial g / \partial u)(u, v)=(\partial h / \partial v)(u, v)$. So we can

$$
\begin{aligned}
F(t, x(t), x(t-\tau)) & =p\left(1+\sin ^{2} \frac{2 \tau t}{\pi}\right)\left[\int_{0}^{x(t-\tau)} s\left(x(t)^{2}+s^{2}\right)^{(p / 2)-1} d s+\int_{0}^{x(t)} s^{p / 2} d s\right] \\
& =\left(1+\sin ^{2} \frac{2 \tau t}{\pi}\right)\left(x(t)^{2}+x(t-\tau)^{2}\right)^{p / 2} .
\end{aligned}
$$

It is obvious that assumptions $\left(A_{1}\right) \sim\left(A_{3}\right)$ and the following $\left(A_{4}^{\prime}\right) \sim\left(A_{6}^{\prime}\right)$ hold: 
$\left(A_{4}^{\prime}\right) x=(x(t), x(t-\tau)) \rightarrow F(t, x(t), x(t-\tau))$ is a continuously differentiable and strictly convex function and also satisfies

$$
F(t, 0,0)=0, \quad F_{1}^{\prime}(t, 0,0)=F_{2}^{\prime}(t, 0,0)=0 \quad \forall t \in[0,2 \gamma \tau]
$$

$\left(A_{5}^{\prime}\right)$ for $\alpha_{2}=1 / p<1 / 2$, there exist constants $M, C>0$, such that when $|x|=$ $\sqrt{x^{2}(t)+x^{2}(t-\tau)} \geq C$ we have

$$
\begin{gathered}
F(t, x(t), x(t-\tau)) \leq \alpha_{2}\left[F_{1}^{\prime}(t, x(t), x(t-\tau)) x(t)+F_{2}^{\prime}(t, x(t), x(t-\tau)) x(t-\tau)\right], \\
F(t, x(t), x(t-\tau)) \leq M|x|^{1 / \alpha_{2}}
\end{gathered}
$$

$\left(A_{6}^{\prime}\right)$

$$
\lim _{|x| \rightarrow 0} \frac{F(t, x(t), x(t-\tau))}{|x|^{2}}=0 .
$$

Thus, (6.6) has at least one nontrivial weak $2 \gamma \tau$-periodic solution by Theorem 5.1.

\section{Acknowledgments}

The project is supported by NNSF of China (10771055), Doctoral Fund of Ministry of Education of China (200805321017).

\section{References}

[1] J. R. Claeyssen, "The integral-averaging bifurcation method and the general one-delay equation," Journal of Mathematical Analysis and Applications, vol. 78, no. 2, pp. 429-439, 1980.

[2] J. L. Massera, "The existence of periodic solutions of systems of differential equations," Duke Mathematical Journal, vol. 17, pp. 457-475, 1950.

[3] T. Yoshizawa, Stability Theory by Liapunov's Second Method, The Mathematical Society of Japan, 1966.

[4] J. L. Kaplan and J. A. Yorke, "Ordinary differential equations which yield periodic solutions of differential delay equations," Journal of Mathematical Analysis and Applications, vol. 48, pp. 317-324, 1974.

[5] R. B. Grafton, "A periodicity theorem for autonomous functional differential equations," Journal of Differential Equations, vol. 6, pp. 87-109, 1969.

[6] R. D. Nussbaum, "Periodic solutions of some nonlinear autonomous functional differential equations," Annali di Matematica Pura ed Applicata, vol. 10, pp. 263-306, 1974.

[7] J. Mawhin, "Equivalence theorems for nonlinear operator equations and coincidence degree theory for some mappings in locally convex topological vector spaces," Journal of Differential Equations, vol. 12, pp. 610-636, 1972. 


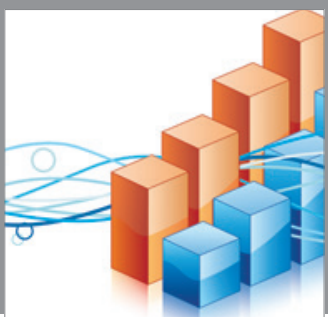

Advances in

Operations Research

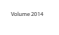

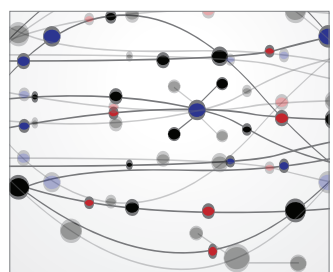

\section{The Scientific} World Journal
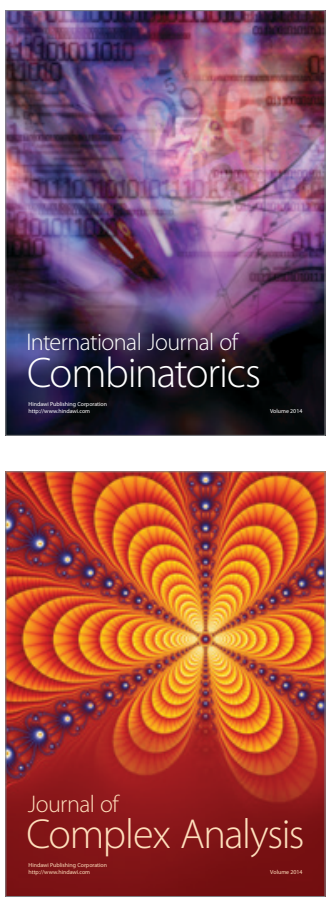

International Journal of

Mathematics and

Mathematical

Sciences
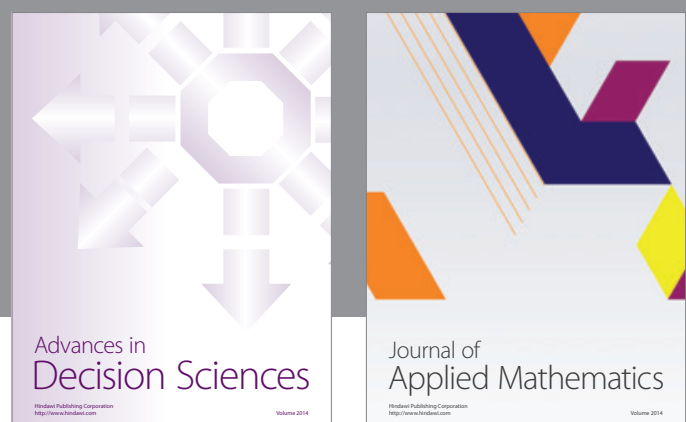

Journal of

Applied Mathematics
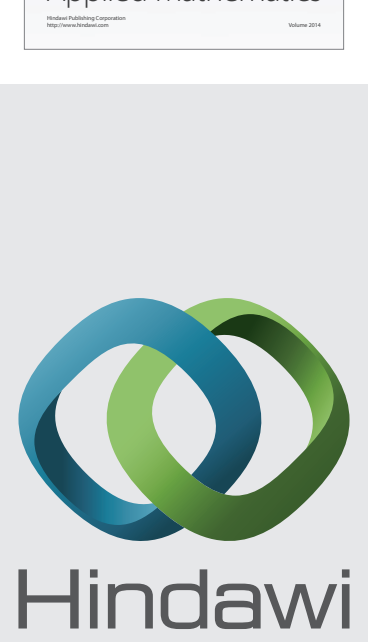

Submit your manuscripts at http://www.hindawi.com
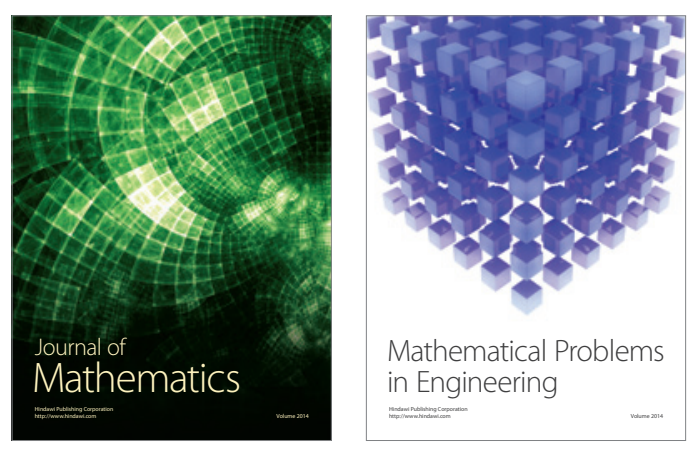

Mathematical Problems in Engineering
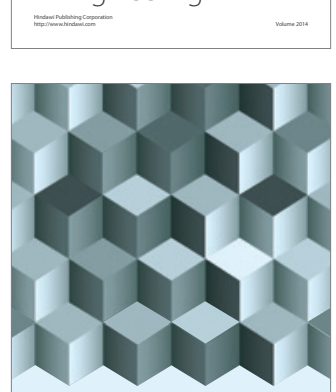

Journal of

Function Spaces
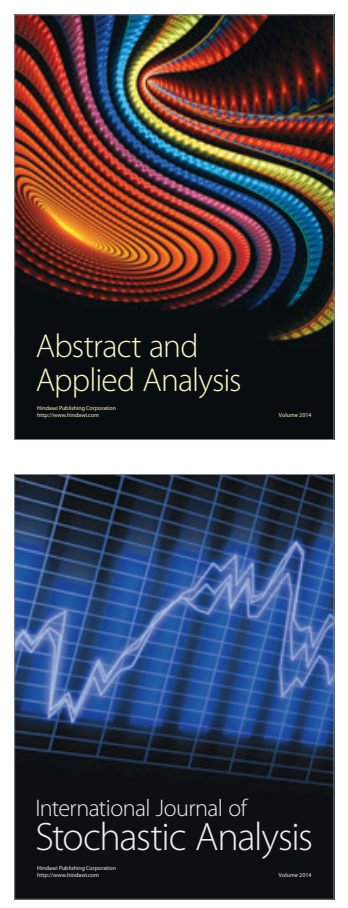

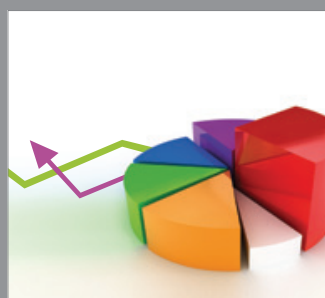

ournal of

Probability and Statistics

Promensencen
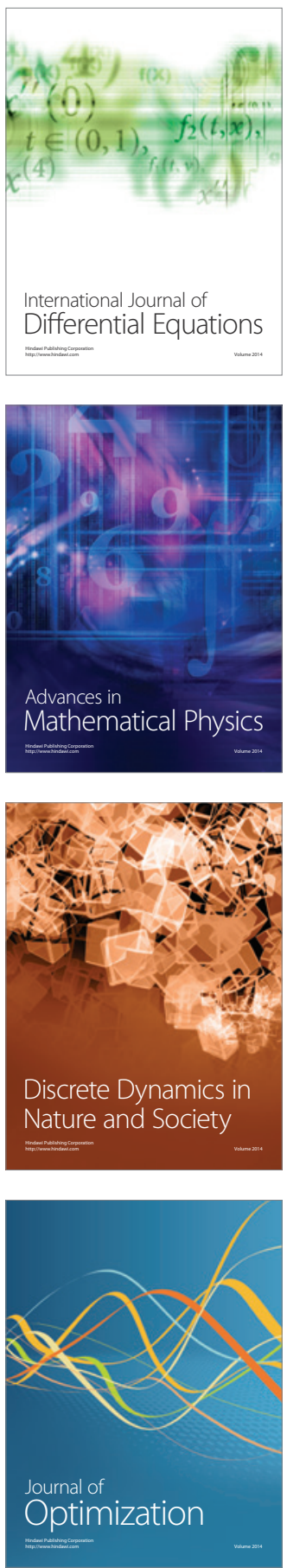